\title{
The Interaction of Inflammatory Markers and Alcohol-Use on Cognitive Function in Korean Male Firefighters
}

\author{
Ji-Ae Yun ${ }^{1,2}$, Kyoung Sook Jeong ${ }^{3}$, Yeon-Soon $\mathrm{Ahn}^{3}$, Yuri Han4, and Kyeong-Sook Choi ${ }^{1,2} \bowtie$ \\ ${ }^{1}$ Department of Neuropsychiatry, Eulji University School of Medicine, Daejeon, Republic of Korea \\ ${ }^{2}$ Department of Neuropsychiatry, Eulji University Hospital, Daejeon, Republic of Korea \\ ${ }^{3}$ Department of Preventive Medicine, Institute Occupational and Environmental Medicine, Yonsei University Wonju College of Medicine, Wonju, \\ Republic of Korea \\ ${ }^{4}$ Yonsei University Wonju College of Medicine, Wonju, Republic of Korea
}

\begin{abstract}
Objective Cognitive functions have been shown to become impaired due to alcoholism. Recently, neuroinflammation gained attention for playing a role in the neurotoxic effect of alcohol. However, there is limited data on the relationship between alcohol and cognitive function, based on the mechanism of inflammation. This study examined whether the interaction between alcohol use and pro-inflammatory biomarkers is related to cognitive function in Korean male firefighters.

Methods A total of 474 firefighters were assessed for alcohol-related problems using CAGE, cognitive functions, and pro-inflammatory biomarkers (CRP, IL-6, TNF- $\alpha$ ). Sequential multiple regression analyses were conducted to determine if inflammatory markers moderate the relationship between alcohol use and cognitive function.

Results Only a decreased attentional function was associated with the interaction of alcohol use and inflammatory markers, after controlling for age, sex, body mass index, lipid profiles, smoking, depression, fatigue, self-reported hypertension, diabetes, and musculoskeletal problems.

Conclusion This study revealed that the interaction between alcohol use and inflammation is related to attentional function in Korean male firefighters. Additionally, this cross-sectional study suggests that diminishing attention, related to alcohol use, may be based on the mechanism of inflammation.

Psychiatry Investig 2021;18(3):205-213
\end{abstract}

Key Words Alcohol dependence, Attention, Cognitive decline, Firefighter, Inflammatory marker.

\section{INTRODUCTION}

Cognitive function is important when performing highly demanding tasks in rapidly changing environments, as experienced by firefighters. ${ }^{1}$ Firefighters require the ability to maintain high levels of attention for prolonged periods; even the smallest mistake while performing procedures could lead to failure on a huge level. ${ }^{2}$

However, firefighters are mostly exposed to a wide variety of physical, chemical, and mental hazards while performing their duties, and many studies have found that these conditions are

Received: March 18, 2020 Revised: November 19, 2020

Accepted: November 20, 2020

$\triangle$ Correspondence: Kyeong-Sook Choi, MD, PhD

Department of Neuropsychiatry, Eulji University Hospital, 95 Dunsanseo-ro, Seo-gu, Daejeon 35233, Republic of Korea

Tel: +82-42-611-3443, Fax: +82-42-611-3445, E-mail: cksinj@eulji.ac.kr

(a) This is an Open Access article distributed under the terms of the Creative Commons Attribution Non-Commercial License (https://creativecommons.org/licenses/bync/4.0) which permits unrestricted non-commercial use, distribution, and reproduction in any medium, provided the original work is properly cited. associated with cognitive decline in firefighters. ${ }^{2-4}$ Further, alcohol-associated cognitive decline has been extensively studied in other populations, ${ }^{5-7}$ and studies have shown that alcohol use is an important factor of cognitive decline in people. Many studies have recorded that the consumption of alcohol by firefighters is higher than that in the general population. ${ }^{8,9}$ Therefore, high alcohol use is also an important factor of cognitive decline among firefighters.

The brain is a major site that exhibits the neurotoxic effects of alcohol: heavy alcohol consumption induces selective neuronal loss as well as reduction of synaptic complexity in specific regions of the brain. Such alcohol-induced changes may precipitate various neurological dysfunctions such as working memory, attention control, depression, anxiety, and executive functions like response inhibition. ${ }^{10,11}$ However, the mechanisms underlying the neurotoxic effects of alcohol on the human brain, and cognitive impairment, are poorly understood. Recently, neuroinflammation gained attention for playing a role in the neurotoxic effect of alcohol. There is growing evi- 
dence of neuroinflammation with increased activation of microglia, and elevated expression of central and peripheral inflammatory factors, in adults with a history of alcohol abuse. ${ }^{12-14}$

Pre-clinical models of alcohol dependence demonstrate microglial activation and expression of inflammatory mediators such as tumor necrosis factor- $\alpha$ (TNF- $\alpha$ ) and interleukin- 6 (IL-6), both in the brain and peripheral blood. ${ }^{15,16}$ In humans, similar processes mediated by a number of inflammatory cytokines and chemokines, including TNF- $\alpha$, IL-6, monocyte chemoattractant protein 1 (MCP1), and IL1 $\beta,^{4,12,17-19}$ may result in cytotoxic effects, thereby impacting neurotransmission, neuroendocrine function, and neural plasticity; $;^{20,21}$ presumably, neuroinflammation also, at least partly, contributes to cognitive deficits linked to alcohol consumption. ${ }^{5}$

Previous studies, which examined the relationship between alcohol use, inflammatory markers, and phenomenologically represented cognitive decline, showed that in alcohol-dependent participants, the level of inflammation was higher than in healthy controls, and that the level of cognitive decline was higher. ${ }^{10,13,22,23}$ However, the study sample was very small, and most subjects were alcohol-dependent patients in the acute withdrawal period. Additionally, the three variables were not simultaneously considered in the analyses in all the previous studies.

In this study, we aimed to investigate the decline of cognitive functions mediated by the neuroinflammation of alcohol by identifying the association of alcohol, inflammatory markers, and cognitive functions in male firefighters in Korea. And then, using moderation analysis, we aimed to determine whether an increase in inflammatory markers in the relationship between alcohol and cognitive functions in firefighters has a moderating effect.

Based on existing studies, we assumed that those who were screened as having alcohol dependence, among Korean male firefighters who are currently performing occupational functions, would also show a decrease in cognitive functions and would show an increase in systemic inflammatory markers. Furthermore, we hypothesized that the interaction between alcohol and inflammation would intensify the association with declined cognitive functions, than alcohol-only main effect.

\section{METHODS}

\section{Study design and ethics}

This study had a cross-sectional study design and was approved by the Institutional Review Board of Dongguk University Ilsan Hospital (DUIH 2017-08-014-001) and Yonsei University Wonju Severance Christian Hospital (IRB No. CR318031). The study was conducted according to the tenets of the Declaration of Helsinki and its revisions. All participants consent- ed in writing to participate in the study after being informed of the nature of the study and its benefits and potential risks.

\section{Participants}

From October 2017 to May 2018, a panel of 516 firefighters from eight fire stations in South Korea was constructed. Among them, we selected only male firefighters to control for the influence of gender diversity. Of 474 male firefighters, we excluded those with C-reactive protein (CRP) concentrations suggestive of acute inflammation and related bacterial infection $(>10 \mathrm{mg} / \mathrm{L})^{24}(\mathrm{n}=9,2 \%)$. Participants whose blood tests and CAGE were not conducted were excluded $(n=34)$. Finally, 431 subjects were included in the analysis.

\section{Measure}

\section{Cognitive functions}

All examinations were conducted in the morning, when work started, in the week of daytime shifts. Cognitive function was tested using a computerized test battery [CNS Vital Signs (CNSVS); Morrisville, NC, USA]. CNSVS is a computerized neurocognitive screening battery comprising seven neuropsychological tests: verbal and visual memory, finger tapping, symboldigit coding, stroop test, shifting attention test, and continuous performance test (CPT). The test takes approximately $20 \mathrm{~min}$ to complete. The tests in the Vital Signs battery are highly reliable, and their concurrent validity has been established by comparing their performance with that of conventional neuropsychological tests on computerized tests. ${ }^{25}$ The validity of each score was evaluated according to the "Validity Indicator" criterion defined in CNSVS. Individual functional tests and cognitive domains computed by raw scores on these tests are listed in Table 1. Raw scores on these individual tests were used to calculate cognitive domain scores, which were used in statistical analysis.

\section{Pro-inflammatory markers: CRP, IL-6, TNF- $\alpha$}

Pro-inflammatory biomarkers of CRP, IL- 6 , and TNF- $\alpha$ were measured using intravenous blood samples. Venous blood was collected directly after the cognitive function test. If the blood test and the cognitive function test could not be performed on the same day, the blood test was performed the day after the cognitive function test. Analyses of biomarker concentrations were performed for all participants simultaneously.

High-sensitivity plasma levels of CRP were measured in duplicate by ELISA based on purified protein and polyclonal anti-CRP antibodies (N-Assay LA CRP-s, Nittobo, Japan). IL-6 (Human IL-6 Immunoassay HS600B, R\&D Systems, USA) and TNF- $\alpha$ (Human TNF- $\alpha$ Immunoassay HSTA00E, R\&D Systems, USA) levels were measured in duplicate by a high sen- 
Table 1. Formulas for calculating the neurocognitive domain scores

\begin{tabular}{lll}
\hline \multicolumn{1}{c}{$\begin{array}{c}\text { BRIEF-CORE } \\
\text { clinical domains }\end{array}$} & \multicolumn{1}{c}{ Corresponding test } & Domain Score Calculations \\
\hline Verbal memory & Verbal memory test (VBM) & $\begin{array}{c}\text { VBM Correct Hits Immediate + VBM Correct Passes Immediate + VBM Correct } \\
\text { Hits Delay + VBM Correct Passes Delay }\end{array}$ \\
Visual memory & Visual memory test (VIM) & $\begin{array}{c}\text { VIM Correct Hits Immediate + VIM Correct Passes Immediate + VIM Correct Hits } \\
\text { Delay + VIM Correct Passes Delay }\end{array}$ \\
Psychomotor speed & Finger Tapping (FTT), & FTT Right Taps Average + FTT Left Taps Average + SDC Correct Responses \\
& Symbol Digit Coding (SDC) & \\
Reaction time & Stroop Test (ST) & (ST Complex Reaction Time Correct + Stroop Reaction Time Correct) / 2 \\
Complex attention & Stroop Test (ST), Shifting & Stroop Commission Errors + SAT Errors + CPT Commission Errors + CPT \\
& Attention (SAT), Continuous & Omission Errors \\
Cognitive flexibility & Performance (CPT) & \\
Stroop Test (ST), Shifting & SAT Correct Responses - SAT Errors - Stroop Commission Errors \\
Processing speed & Attention (SAT) & \\
Executive function & Shifting Attention (SAT) & SAT Correct Responses - SAT Errors \\
Simple attention & Continuous Performance (CPT) & Continuous Performance (CPT) Correct Responses minus CPT Commission Errors \\
Motor speed & Finger Tapping (FTT) & Finger Tapping Test Right Taps Average + Finger Tapping Test Left Taps Average \\
\hline
\end{tabular}

sitivity enzyme-linked immunosorbent assay (ELISA).

\section{Screening for alcohol dependence: CAGE}

The CAGE questionnaire ${ }^{26}$ is one of the most widely researched of all alcohol screening tools, ${ }^{27}$ and because of its brevity and ease of administration, it may also be the most widely employed. ${ }^{28}$ In a study examining the sensitivity and specificity of CAGE in Koreans, a score of 3 was recommended as the cut off point for strictly selecting alcohol dependence. In addition, CAGE score of more than 3 was known to have a higher specificity for alcohol dependence. ${ }^{29}$ In this study, participants obtaining a CAGE score of more than 3 were classified under the alcohol dependence group. ${ }^{30}$

\section{Potential confounders}

Potential confounders that are known to play a role in both cognitive functioning and systemic levels of inflammatory markers ${ }^{5,31}$ that were utilized in the study were age; smoking status (never/stopped smoker/current smoker); body mass index; lipid profiles (total cholesterol, triglyceride, LDL-cholesterol); and self-reported presence/absence of diagnosed hypertension, diabetes, and unspecified musculoskeletal problems. Self-rated depressive symptoms that evaluated with Patient Health Questionnaire-9 (PHQ-9) ${ }^{32}$ and fatigue symptoms that evaluated with Fatigue Severity Scale (FSS) ${ }^{33}$ at the time of testing were also included in the study as potential confounders.

\section{Analyses}

All statistical analyses were performed using SPSS 21.0 (IBM Corp., Armonk, NY, USA). Interval scaled data were described as means, and standard deviation (SD) and nominal variables by frequency and valid percent. We divided the participants into two groups according to their CAGE score (i.e., groups with a CAGE score of 3 or higher and those without). Independent sample t-tests were used to compare quantitative demographic data and psychiatric scale score between the two groups. A chi-square test was used to compare qualitative variables.

The distributions for IL-6, TNF- $\alpha$, and CRP were positively skewed and therefore, these variables were log-transformed to better conform to a normal distribution.

Composite scores of the inflammatory biomarker levels were computed. These composite scores were computed by first standardizing (z-transforming) each log-transformed biomarker, and then computing the mean of these z-scores. Since the pattern of bivariate correlations between TNF- $\alpha$ and the outcome measures differed from that of the other biomarkersbivariate correlations between TNF- $\alpha$ and attention were absent, while the other biomarkers (IL-6, and CRP) were negatively correlated with the attention-a biomarker composite score excluding TNF- $\alpha$ was also computed for the analyses.

The linear regression analysis was used to determine whether alcohol and inflammatory markers were related to each cognitive domain. In the adjusted model, age, smoking, BMI, lipid profiles (total cholesterol, triglyceride, LDL-cholesterol), diagnosed diseases (hypertension, diabetes, and unspecified musculoskeletal problems), depressive symptoms, and fatigue were included as confounder variables.

Sequential multiple regression analyses were conducted to determine if inflammatory markers moderated the relationship between alcohol dependence and cognitive function. To exam- 
ine this, we created the interaction term of alcohol (CAGE $\geq 3$ ) and each inflammatory marker.

For dependent measure (each cognitive domain), regression analyses were performed as follows:

Step 1: potential confounders mentioned above,

Step 2: variables included in Step 1; alcohol (CAGE $\geq 3$ ), one of the inflammatory markers or composite score,

Step 3: variables included in Step 1 and 2, interaction term of alcohol dependence and one of the inflammatory markers or composite score (Alcohol $\times$ Inflammatory marker).

ModGraph $-\mathrm{I}^{34}$ was used to visually illustrate this interaction.

\section{RESULTS}

\section{Descriptive statistics}

Sample characteristics are presented in Table 2. Seventy-three participants (16.9\% of the total) had a CAGE score of 3 or more.

Table 2. The sociodemographic characteristics and pro-inflammatory markers of the total samples

\begin{tabular}{|c|c|c|}
\hline $\mathrm{N}=431$ & $\operatorname{Mean}(\mathrm{N})$ & $\mathrm{SD}(\%)$ \\
\hline Age & 40.84 & 8.83 \\
\hline \multicolumn{3}{|l|}{ Marriage } \\
\hline Single & 112 & 25.9 \\
\hline Married & 316 & 73.0 \\
\hline Divorced & 5 & 1.2 \\
\hline \multicolumn{3}{|l|}{ Smoking } \\
\hline Never & 144 & 40.2 \\
\hline Stop smoker & 155 & 43.3 \\
\hline Current smoker & 131 & 36.6 \\
\hline Body mass index & 24.38 & 2.33 \\
\hline \multicolumn{3}{|l|}{ Lipid profiles } \\
\hline Total cholesterol & 189.71 & 34.00 \\
\hline Triglyceride & 169.64 & 101.27 \\
\hline LDL-cholesterol & 118.95 & 30.98 \\
\hline Depression (PHQ9) & 2.17 & 3.00 \\
\hline Fatigue (FSS) & 24.28 & 11.55 \\
\hline \multicolumn{3}{|l|}{ Diagnosed disease } \\
\hline Hypertension & 71 & 19.8 \\
\hline Diabetes & 72 & 20.1 \\
\hline Musculoskeletal problems & 70 & 19.6 \\
\hline \multicolumn{3}{|l|}{ Pro-inflammatory markers } \\
\hline $\mathrm{CRP}(\mathrm{mg} / \mathrm{L})$ & 1.00 & 1.33 \\
\hline IL-6 (pg/mL) & 1.17 & 1.04 \\
\hline TNF- $\alpha(p g / m L)$ & 0.77 & 0.30 \\
\hline
\end{tabular}

PHQ9: Patient Health Questionnaire-9, FSS: Fatigue Severity Scale, CRP: C-reactive protein, IL-6: interleukin-6, TNF- $\alpha$ : tumor necrosis factor- $\alpha$
There were no significant differences between $\mathrm{CAGE} \geq 3$ and $\mathrm{CAGE}<3$ participants, except the smoking status.

\section{Associated factor of each domain of cognitive function (Table 3)}

Alcohol use (CAGE $\geq 3$ ) was not associated with all cognitive functions in the unadjusted model, but only with decreased attention in the adjusted model. IL-6 was associated with decreased executive function and attention. But after controlling for confounder variables, only IL- 6 was associated with decreased attention. CRP was associated with a reduction in psychomotor speed and motor speed only in the adjusted model. TNF- $\alpha$ was associated with a reduction in psychomotor speed and processing speed only in the adjusted model. The 2-biomarker composite, IL-6 and CRP (comp2), was associated with a decrease in psychomotor speed and executive function; however, in the adjusted model, they were only associated with a decrease in attention. The 3-biomarker composite (comp3), which is comp2 plus TNF- $\alpha$, was associated with reduced attention only in the adjusted model.

\section{Moderation analyses using interaction term of alcohol and inflammatory marker (Table 4)}

In the first step of sequential multiple regression for each cognitive domain, all confounder variables were included. In the second step, alcohol and inflammatory markers were added to these variables. The main effects of each inflammatory marker were identified using five regression analyses on IL-6, CRP, TNF- $\alpha$, 2-biomarker composite (IL-6, CRP), and 3-biomarker composite (IL-6, CRP, TNF- $\alpha$ ). In the final step, the interaction term for the association between each inflammatory marker and alcohol was entered to confirm the interaction effect.

In all cognitive domains, except attention, the interaction effect was not significant. In terms of attentional function, the interaction effect of all inflammatory markers except TNF- $\alpha$ with alcohol was significant. Below is a detailed explanation of the interaction analysis for attention.

\section{$\mathrm{CRP} \times$ alcohol}

In the first step, triglyceride $(\mathrm{B}=-0.008, \mathrm{SE}=0.008, \mathrm{p}=0.035)$ and smoking $(\mathrm{B}=0.492, \mathrm{SE}=0.248, \mathrm{p}=0.048)$ were associated with attention. In the second step, there was a significant main effect of alcohol on attention $(B=-0.885, S E=0.322, p=0.007)$ while CRP had no main effect on attention $(B=-0.618, S E=$ $0.322, \mathrm{p}=0.057)$. In the final step, the interaction between alcohol and CRP was significantly associated with attention $(\mathrm{B}=-3.920, \mathrm{SE}=0.922, \mathrm{p}<0.001)$. The final model accounts for $22.7 \%$ of the variance. 


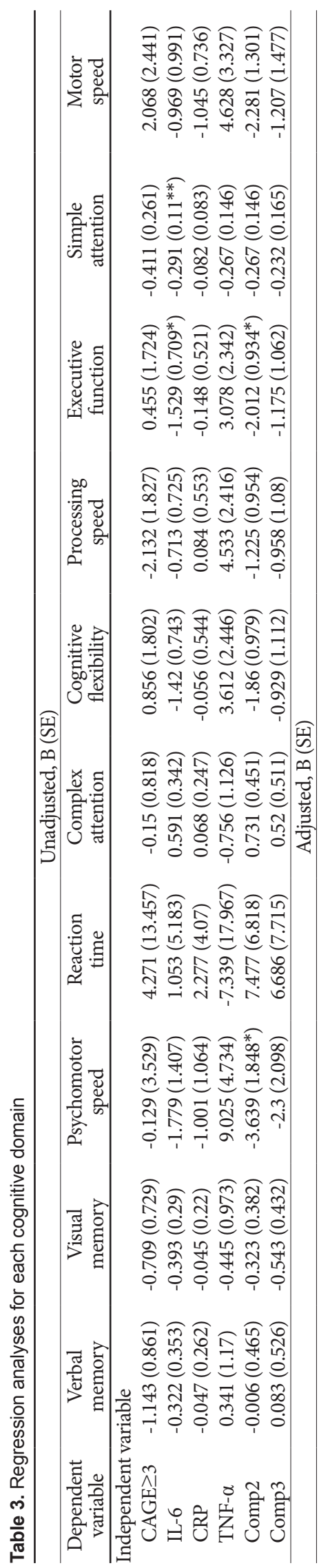

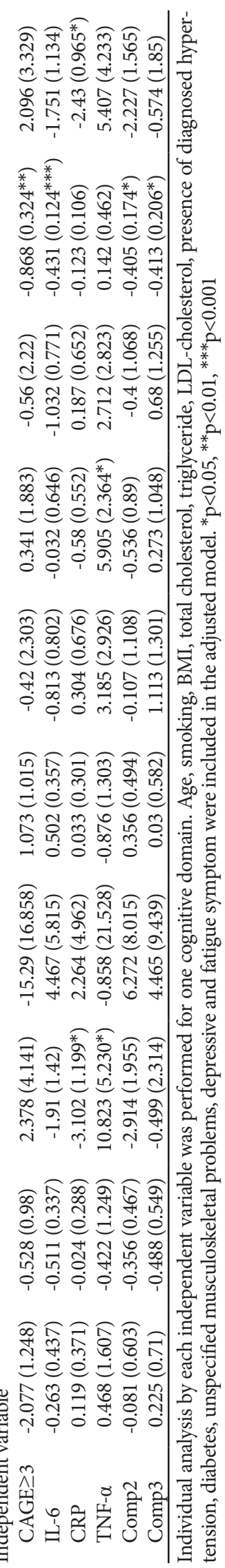

\section{IL-6 $\times$ alcohol}

In the first step, there were no factors associate with attention. In the second step, there was significant main effect of alcohol $(\mathrm{B}=-0.988, \mathrm{SE}=0.343, \mathrm{p}=0.004)$ and IL-6 $(\mathrm{B}=-1.015$, $\mathrm{SE}=0.394, \mathrm{p}=0.011)$ on attention, respectively. In the final step, the interaction between alcohol dependence and IL- 6 was significantly associated with attention $(B=-2.792, S E=1.145$, $\mathrm{p}=0.016$ ). The final model accounts for $21.6 \%$ of the variance.

\section{TNF- $\alpha \times$ alcohol}

In the first step, triglyceride $(\mathrm{B}=-0.003, \mathrm{SE}=0.001, \mathrm{p}=0.035)$ and smoking $(\mathrm{B}=0.492, \mathrm{SE}=0.248, \mathrm{p}=0.048)$ were associated with attention. In the second step, there was a significant main effect of alcohol on attention $(B=-0.878, S E=0.325, p=0.007)$ while TNF- $\alpha$ did not have main effect on attention $(B=-0.474$, $\mathrm{SE}=0.859, \mathrm{p}=0.581$ ). In the final step, the interaction between alcohol and TNF- $\alpha$ was not associated with attention $(B=-6.419$, $\mathrm{SE}=3.642, \mathrm{p}=0.080$ ). The final model accounts for $15.7 \%$ of the variance.

\section{Biomarker composite $\times$ alcohol}

In the second analysis, the composite biomarker measure of IL-6, CRP, and TNF- $\alpha$ was associated with attention, and the composite biomarker measure of IL-6, CRP except TNF- $\alpha$, was also related to attention. Alcohol was also associated with attention in both analyses. In the final step, the interaction between alcohol and both 2- and 3-biomarker composite was associated with attention respectively (2-biomarker composite $\mathrm{B}=-1.675, \mathrm{SE}=0.430, \mathrm{p}<0.001$; 3-biomarker composite $\mathrm{B}=$ $-2.018, \mathrm{SE}=0.520, \mathrm{p}<0.001)$. Each final model accounts for $25.9 \%$ and $25.5 \%$ of the variance. Figure 1 was used to visually illustrate this interaction.

\section{DISCUSSION}

The purpose of the present study was to investigate the potential moderating role of inflammatory processes, in terms of systemic levels of pro-inflammatory markers, in cognitive function, while adjusting for important confounders, in Korean male firefighters.

There was no significant difference in the average score of each cognitive domain in the $\mathrm{CAGE} \geq 3$ group and $\mathrm{CAGE}<3$ groups. Regression analysis also showed no significant relationship between alcohol (CAGE $\geq 3$ ) and the respective cognitive domains. Considering various confounder variables, alcohol use (CAGE $\geq 3$ ) was associated with lower attention.

The same analysis was performed for the subjects who selected the eye-opener item, the 4th item of CAGE that suggests severe withdrawal symptom ${ }^{35}$ out of $\mathrm{CAGE} \geq 3$ group, but the results were the same as the previous analysis. In contrast, half 
Table 4. Sequential multivariate regression analyses for identifying interaction effects result in the factors related to attention in Korean male firefighters

\begin{tabular}{|c|c|c|c|c|c|c|}
\hline & \multicolumn{3}{|c|}{ Main effect analysis } & \multicolumn{3}{|c|}{ Interaction effect analysis } \\
\hline & B & SE & $\mathrm{t}$ & B & SE & $\mathrm{t}$ \\
\hline $\mathrm{CAGE} \geq 3$ & -0.99 & 0.34 & $-2.88^{* *}$ & -1.07 & 0.34 & $-3.14^{* *}$ \\
\hline IL-6 & -1.02 & 0.39 & $-2.57^{*}$ & -0.64 & 0.42 & -1.52 \\
\hline $\mathrm{CAGE} \geq 3 \times \mathrm{IL}-6$ & & & & -2.79 & 1.14 & $-2.44^{*}$ \\
\hline R squared & & & 0.191 & & & 0.216 \\
\hline $\mathrm{CAGE} \geq 3$ & -0.88 & 0.32 & $-2.75^{* *}$ & -1.88 & 0.39 & $-4.85^{* * *}$ \\
\hline CRP & -0.62 & 0.32 & -1.92 & -0.18 & 0.33 & -0.54 \\
\hline $\mathrm{CAGE} \geq 3 \times \mathrm{CRP}$ & & & & -3.92 & 0.92 & $-4.25^{* * *}$ \\
\hline$\underline{\mathrm{R} \text { squared }}$ & & & 0.158 & & & 0.227 \\
\hline $\mathrm{CAGE} \geq 3$ & -0.88 & 0.32 & $-2.70^{* *}$ & -1.94 & 0.68 & $-2.84^{* *}$ \\
\hline TNF- $\alpha$ & -0.47 & 0.86 & -0.55 & -0.10 & 0.88 & -0.11 \\
\hline CAGE $\geq 3 \times$ TNF $-\alpha$ & & & & -6.42 & 3.64 & -1.76 \\
\hline R squared & & & 0.143 & & & 0.157 \\
\hline $\mathrm{CAGE} \geq 3$ & -1.03 & 0.34 & $-3.03^{* *}$ & -1.17 & 0.33 & $-3.54^{* *}$ \\
\hline Comp2 (IL-6, CRP) & -0.44 & 0.15 & $-2.86^{* *}$ & -0.22 & 0.16 & -1.41 \\
\hline CAGE $\geq 3 \times$ Comp 2 & & & & -1.68 & 0.43 & $-3.90^{* * *}$ \\
\hline R squared & & & 0.197 & & & 0.259 \\
\hline $\mathrm{CAGE} \geq 3$ & -1.07 & 0.34 & $-3.13^{* *}$ & -1.31 & 0.34 & $-3.90^{* * *}$ \\
\hline Comp3 (IL-6, CRP, TNF- $\alpha$ ) & -0.49 & 0.18 & $-2.69^{* *}$ & -0.25 & 0.19 & -1.35 \\
\hline CAGE $\geq 3 \times$ Comp 3 & & & & -2.02 & 0.52 & $-3.88^{* * *}$ \\
\hline$\underline{\mathrm{R} \text { squared }}$ & & & 0.193 & & & 0.255 \\
\hline
\end{tabular}

Age, smoking, BMI, total cholesterol, triglyceride, LDL-cholesterol, presence of diagnosed hypertension, diabetes, unspecified musculoskeletal problems, depressive and fatigue symptom were included in all the steps of analyses. ${ }^{*} \mathrm{p}<0.05,{ }^{* *} \mathrm{p}<0.01,{ }^{* * *} \mathrm{p}<0.001$. Comp2: 2 -biomarker composite (IL-6, CRP), Comp3: 3-biomarker composite (IL-6, CRP, TNF- $\alpha$ )

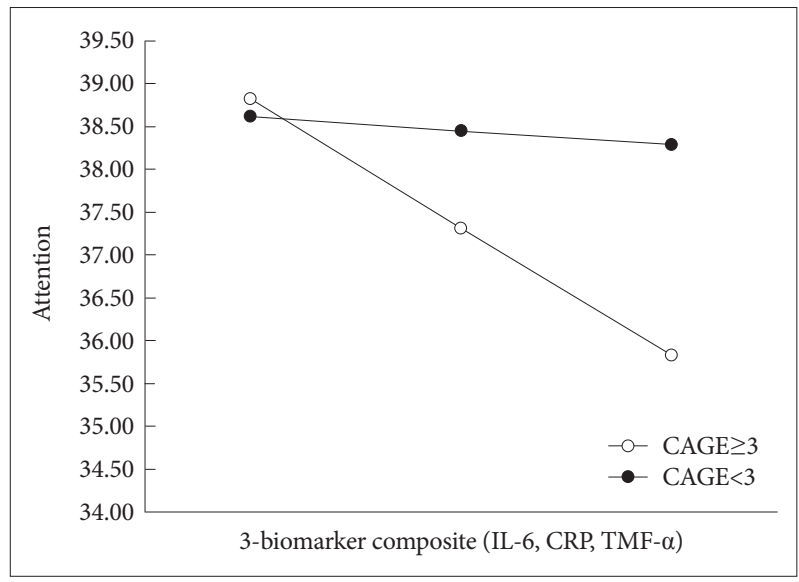

Figure 1. The interaction between alcohol use and 3-inflammatory biomarker composite (IL-6, CRP, TNF- $\alpha$ ) on attention.

of the participants who chose the eye-opener item of CAGE did not belong to the CAGE $\geq 3$ group ( 22 of 46 eye-openers). This may suggest a case that our sample consists of shift workers and uses alcohol as a self-aid for sleep in the morning, even if it is not evaluated as alcohol dependence by the sum of CAGE scores. ${ }^{36,37}$ Additionally, in our sample, depression was not associated with $\mathrm{CAGE} \geq 3$, but fatigue was. In the case of shift workers like our sample, it is necessary to evaluate not only the average level of alcohol but also the patterns of alcohol use. ${ }^{37}$

Meanwhile, in our study, except for attention, CAGE $\geq 3$ was no significant association with the decrease of other cognitive domains. Existing studies have reported various degrees of cognitive decline in alcohol-dependent patients. A hypothesis that has attracted increasing attention suggests that alcoholism is a 'disinhibitory disorder. This would account for poor performance in a variety of cognitive tasks assessing dominant response inhibition in abstinent alcoholics. ${ }^{38}$

However, this does not show consistent results in all studies, ${ }^{39,40}$ which suggests the ceiling effect of the test due to very few errors made by participants in both groups. Since the alcohol-dependent group screened in our study is also a group of working firefighters, this possibility of ceiling effect cannot be ruled out; thus, the difference of cognitive function between the two groups classified by CAGE may not have been evident. Nevertheless, in CPT, the difference of result is clear, meaning that disinhibition is more sensitively detected. In addition, since 
CPT in the battery conducted in our study was performed for 5 minutes shorter than the existing test time, the significant decreasing of $\mathrm{CPT}$ score in $\mathrm{CAGE} \geq 3$ group within the short test time suggests a noticeable decrease in attention compared to other cognitive functions of these groups.

Regression analysis of the relationship between each inflammatory marker and cognitive function showed associations for some cognitive domains including attention. In particular, the adjusted model that controlled the confounder variables showed more associations between inflammatory marker and cognitive function. Uniquely, in addition to attention, the cognitive functions related to some inflammatory markers were limited to the psychomotor speed, processing speed and motor speed. Attention was measured by CPT, and the remaining three cognitive domains related to speed were measured by the finger tapping test (FTT) and symbol digit coding (SDC). Thus, it can be suggested that the decrease in the three test scores of CPT, FTT, and SDC is associated with each inflammatory marker.

Unlike other inflammatory markers, TNF- $\alpha$ showed a protective effect on psychomotor speed and processing speed in the adjusted model. This finding may support a neuroprotective role of TNF- $\alpha$ under immunologically unchallenged conditions, as has been suggested in some previous studies. ${ }^{31,41}$ In one previous study, a significant association between the TNF$\alpha-308 \mathrm{G} \rightarrow$ A polymorphism and processing speed was observed, similar with our results in terms of association between TNF- $\alpha$ and processing speed, as well as psychomotor speed. ${ }^{41}$ In addition, the correlation between inflammatory markers and cognitive functions in $\mathrm{CAGE} \geq 3$ group showed significant results only on attention, and only TNF- $\alpha$ showed no correlation with attention contrary to IL- 6 and CRP. This is the same result from previous studies. ${ }^{31}$ More research is needed on the role of TNF- $\alpha$ in the process of influence of alcohol on cognitive function.

In previous studies of alcohol and inflammation, the subjects were usually alcohol-dependent patients in acute withdrawal period. ${ }^{10,13,22,23}$ Their CRP level in the withdrawal state was shown to be much higher than the ones in this study. Our study excluded those with a CRP of 10 or higher, and differences within the normal range of the CRP had a moderating effect on the relationship between alcohol and attention. CRP is a general marker for inflammation and infection. Although CRP is not a very specific prognostic indicator, it was known to be used as a very rough proxy for, for example, heart disease risk. Arterial damage which resulted from white blood cell invasion and inflammation within the wall cause increasing CRP within subnormal level, which was regarded as a marker for risk of heart disease. ${ }^{42-44}$ Likewise, with regard to our results, the association of attention with an increase in the subnormal level of inflammatory markers would suggest the possibility of neurotoxic effect through low grade inflammatory processes. $^{31}$

Unlike previous studies, this study analyzes the alcohol, cognitive function, and inflammatory marker simultaneously with a relatively large sample of firefighters. As a result, we found that the interaction between alcohol use and inflammation was related to decline their attentional function, after controlling for confounder variables that may affect inflammation and cognitive function. Moreover, interaction effect was greater than the main effect of alcohol on attention. In other cognitive domains, however, these interactions were not shown.

We suggest two possibilities for the lack of associations with other cognitive domains, except attention. First, the results of this study should be interpreted differently from the study of alcohol-dependent patients who visited the hospital, because the participants of our study continued their occupational function, even though they were screened as having alcohol dependence. Participants screened as having alcohol dependence in this study may not yet have significant cognitive impairment, which could be confirmed by the test (total neurocognition index below average in our study; $\mathrm{n}=17$ ). Also, as mentioned earlier, the ceiling effect cannot be excluded because the average age of participants screened as having alcohol dependence is 41 years, and they are firefighters who have intact occupational function.

Second, unlike memory or executive function representing the function of some particular brain region, attention is an indicator of overall brain function. The various models of attention consistently suggest the interaction of cortical (frontal, prefrontal, parietal) with subcortical [limbic system, reticular activating system (RAS), and basal ganglia] structures as well as the pathways/projections between the basal ganglia, thalamus, and frontal lobes to form a complex functional system. ${ }^{45}$ The effect of alcohol and inflammation was only limited to reduced attention and might reflect an intermediate phenotype of cognitive impairment, which means cognitive change is not yet evident. Based on our findings, attentional functioning seems to be the cognitive domain that is the most sensitive to low grade inflammation, in terms of systemic levels of IL-6, CRP. It is suggested that inflammatory responses are an important factor in the influence of alcohol on a wide range of brain areas related to attention.

The present study has several limitations. First, the crosssectional design limits its ability to establish causal relationships. Therefore, the results should be interpreted cautiously, and future longitudinal and experimental research is required. Second, decreased cognitive function and increased inflammatory markers can be affected by alcohol use as well as comorbid psychiatric disorders. ${ }^{46,47}$ In our analysis, depressive symptoms and fatigue were included in the adjusted model as confounder variables, but other factors such as burnout ${ }^{48,49}$ and 
$\mathrm{PTSD}^{50}$ need to be considered. Furthermore, this study relied solely on self-reported questionnaires for assessing the alcohol dependence and other psychiatric covariates. Although all the scales used in this study are well validated, future studies using standardized interviews or observational methods could provide more accurate and detailed information on the complex mechanisms underlying the relationship between cognitive functions, inflammatory process and other psychiatric symptoms. Third, the use of antibiotics or non-steroidal antiinflammatory drugs, either during the course of the study or within the month prior to enrollment, was not considered in the analyses. Although we investigated the self-reported several diagnosed diseases, we could not consider all the physiological diseases such as rheumatic arthritis, liver cirrhosis, or other inflammatory diseases. Additionally, alcohol dependence is associated with several other lifestyle and clinical characteristics. The present study did not analyze such changes in lifestyle and quality of life, including financial problems or any recent stressful events that could have influenced cytokine production. Therefore, while the analysis considered many potential confounders, a residual confounding effect cannot be completely excluded. Fourth, all of the participants were male, and caution must therefore be applied when extrapolating to populations including both sexes. Comparative future studies on female firefighters should, therefore, be conducted.

Even with these limitations, the present study contributes new empirical data and knowledge. There has been a lack of studies investigating systemic inflammatory markers and alcohol use among people who are relatively healthy and working, and this study included a broad range of cognitive functions via multiple neurocognitive function tests to contribute to the field. In addition, it has been repeatedly confirmed that refraining from consuming alcohol may be an important factor in preventing a decrease in cognitive function, in particular, lowering concentration or attention, among firefighters.

We examined the moderating effect of inflammatory marker between alcohol use and cognitive decline in Korean male firefighters. As a result, the interaction between alcohol and inflammation (CRP, IL-6) was associated with decreased attentional function. In particular, the degree of this association was much greater than main effect of alcohol alone. However, no association was found in other cognitive domains. This study identified the moderating effects of inflammation between alcohol and attention in subnormal levels in a relatively healthy sample. In addition, it has been repeatedly confirmed that refraining from using alcohol may be an important factor in preventing a decrease in the cognitive function of firefighters, in particular, the lowering of concentration or diminishing attention.

\section{Acknowledgments}

This work was supported by Korea Environment Industry \& Technology Institute (KEITI) through The Chemical Accident Prevention Technology Development Program (or Project), funded by Korea Ministry of Environment (MOE) (2017001970001).

\section{Conflicts of Interest}

The authors have no potential conflicts of interest to disclose.

\section{Author Contributions}

Conceptualization: Kyoung Sook Jeong, Yeon-Soon Ahn. Data curation: Kyoung Sook Jeong, Yeon-Soon Ahn. Formal analysis: Ji-Ae Yun, Yuri Han. Funding acquisition: Yeon-Soon Ahn. Investigation: Kyoung Sook Jeong, Yeon-Soon Ahn. Methodology: Kyoung Sook Jeong, Yeon-Soon Ahn, Kyeong-Sook Choi. Supervision: Kyeong-Sook Choi. Writing-original draft: Ji-Ae Yun. Writing_-review \& editing: Kyeong-Sook Choi, Ji-Ae Yun.

\section{ORCID iDs}

$\begin{array}{ll}\text { Ji-Ae Yun } & \text { https://orcid.org/0000-0002-6682-3275 } \\ \text { Kyoung Sook Jeong } & \text { https://orcid.org/0000-0002-6897-8289 } \\ \text { Yeon-Soon Ahn } & \text { https://orcid.org/0000-0002-0039-069X } \\ \text { Yuri Han } & \text { https://orcid.org/0000-0002-4618-2546 } \\ \text { Kyeong-Sook Choi } & \text { https://orcid.org/0000-0003-3933-7121 }\end{array}$

\section{REFERENCES}

1. Walker A, Argus C, Driller M, Rattray B. Repeat work bouts increase thermal strain for Australian firefighters working in the heat. Int J Occup Environ Health 2015;21:285-293.

2. Kujawski S, Słomko J, Tafil-Klawe M, Zawadka-Kunikowska M, Szrajda J, Newton JL, et al. The impact of total sleep deprivation upon cognitive functioning in firefighters. Neuropsychiatr Dis Treat 2018;14: 1171-1181.

3. Hemmatjo R, Motamedzade M, Aliabadi M, Kalatpour O, Farhadian $\mathrm{M}$. The effect of artificial smoke compound on physiological responses, cognitive functions and work performance during firefighting activities in a smoke-diving room: an intervention study. Int J Occup Saf Ergon 2018;24:358-365.

4. Bell S, Mehta G, Moore K, Britton A. Ten-year alcohol consumption typologies and trajectories of C-reactive protein, interleukin- 6 and interleukin-1 receptor antagonist over the following 12 years: a prospective cohort study. J Intern Med 2017;281:75-85.

5. Coppens V, Morrens M, Destoop M, Dom G. The interplay of inflammatory processes and cognition in alcohol use disorders-a systematic review. Front Psychiatry 2019;10:632.

6. Sabia S, Elbaz A, Britton A, Bell S, Dugravot A, Shipley M, et al. Alcohol consumption and cognitive decline in early old age. Neurology 2014;82:332-339.

7. Topiwala A, Allan CL, Valkanova V, Zsoldos E, Filippini N, Sexton C, et al. Moderate alcohol consumption as risk factor for adverse brain outcomes and cognitive decline: longitudinal cohort study. BMJ 2017; 357:j2353.

8. KOSIS. Socio-Demographic Distribution of Alcohol Use Disorders 1-Year Prevalence. Daejeon: Ministry of Health and Welfare Korea; 2016.

9. Kim MG, Seo JI, Kim K, Ahn YS. Nationwide firefighter survey: the prevalence of lower back pain and its related psychological factors among Korean firefighters. Int J Occup Saf Ergon 2017;23:447-456.

10. Hanak C, Benoit J, Fabry L, Hein M, Verbanck P, de Witte P, et al. Changes in pro-inflammatory markers in detoxifying chronic alcohol abusers, divided by lesch typology, reflect cognitive dysfunction. Alcohol Alcohol 2017;52:529-534.

11. Kopera M, Wojnar M, Brower K, Glass J, Nowosad I, Gmaj B, et al. Cognitive functions in abstinent alcohol-dependent patients. Alcohol 
2012;46:665-671.

12. Achur RN, Freeman WM, Vrana KE. Circulating cytokines as biomarkers of alcohol abuse and alcoholism. J Neuroimmune Pharmacol 2010;5:83-91.

13. Wilhelm CJ, Fuller BE, Huckans M, Loftis JM. Peripheral immune factors are elevated in women with current or recent alcohol dependence and associated with altered mood and memory. Drug Alcohol Depend 2017;176:71-78.

14. He J, Crews FT. Increased MCP-1 and microglia in various regions of the human alcoholic brain. Exp Neurol 2008;210:349-358.

15. Qin L, He J, Hanes RN, Pluzarev O, Hong JS, Crews FT. Increased systemic and brain cytokine production and neuroinflammation by endotoxin following ethanol treatment. J Neuroinflammation 2008;5:10.

16. Kalk NJ, Guo Q, Owen D, Cherian R, Erritzoe D, Gilmour A, et al. Decreased hippocampal translocator protein $(18 \mathrm{kDa})$ expression in alcohol dependence: a [11C]PBR28 PET study. Transl Psychiatry 2017; 7:e996.

17. Donzis EJ, Tronson NC. Modulation of learning and memory by cytokines: signaling mechanisms and long term consequences. Neurobiol Learn Mem 2014;115:68-77.

18. Prieto GA, Cotman CW. Cytokines and cytokine networks target neurons to modulate long-term potentiation. Cytokine Growth Factor Rev 2017;34:27-33.

19. King JA, Nephew BC, Choudhury A, Poirier GL, Lim A, Mandrekar P. Chronic alcohol induced liver injury correlates with memory deficits: role for neuroinflammation. Alcohol 2020;83:75-81.

20. Beumer W, Gibney SM, Drexhage RC, Pont-Lezica L, Doorduin J, Klein HC, et al. The immune theory of psychiatric diseases: a key role for activated microglia and circulating monocytes. J Leukoc Biol 2012; 92:959-975.

21. Miller AH, Maletic V, Raison CL. Inflammation and its discontents: the role of cytokines in the pathophysiology of major depression. Biol Psychiatry 2009;65:732-741.

22. Yen CH, Ho PS, Yeh YW, Liang CS, Kuo SC, Huang CC, et al. Differential cytokine levels between early withdrawal and remission states in patients with alcohol dependence. Psychoneuroendocrinology 2017; 76:183-191.

23. Leclercq S, Cani PD, Neyrinck AM, Stärkel P, Jamar F, Mikolajczak M, et al. Role of intestinal permeability and inflammation in the biological and behavioral control of alcohol-dependent subjects. Brain Behav Immun 2012;26:911-918.

24. Biasucci LM. CDC/AHA Workshop on markers of inflammation and cardiovascular disease: application to clinical and public health practice: clinical use of inflammatory markers in patients with cardiovascular diseases: a background paper. Circulation 2004;110:e560-e567.

25. Gualtieri CT, Johnson LG. Reliability and validity of a computerized neurocognitive test battery, CNS Vital Signs. Arch Clin Neuropsychol 2006;21:623-643.

26. Ewing JA. Detecting alcoholism. The CAGE questionnaire. JAMA 1984;252:1905-1907.

27. Maisto SA, Connors GJ, Allen JP. Contrasting self-report screens for alcohol problems: a review. Alcohol Clin Exp Res 1995;19:1510-1516.

28. Shields AL, Caruso JC. A reliability induction and reliability generalization study of the CAGE questionnaire. Educ Psychol Meas 2004;64: 254-270.

29. Magruder-Habib K, Stevens HA, Alling WC. Relative performance of the MAST, VAST, and CAGE versus DSM-III-R criteria for alcohol dependence. J Clin Epidemiol 1993;46:435-441.

30. Park BK, Kim JS, Lee DB. Comparison of four alcoholism screening tools based on sensitivity and specificity for DSM-IV criteria. J Korean Acad Fam Med 2000;21:1427-1434.

31. Stenfors CUD, Jonsdottir IH, Magnusson, Hanson LL, Theorell T. Associations between systemic pro-inflammatory markers, cognitive function and cognitive complaints in a population-based sample of working adults. J Psychosom Res 2017;96:49-59.

32. Spitzer RL, Kroenke K, Williams JB. Validation and utility of a self-report version of PRIME-MD: the PHQ primary care study. Primary Care Evaluation of Mental Disorders. Patient Health Questionnaire. JAMA 1999;282:1737-1744.

33. Krupp LB, LaRocca NG, Muir-Nash J, Steinberg AD. The fatigue severity scale: application to patients with multiple sclerosis and systemic lupus erythematosus. Arch Neurol 1989;46:1121-1123.

34. Jose PE. ModGraph-I: A programme to compute cell means for the graphical display of moderational analyses: the internet version, Version 3.0. Victoria University of Wellington, Wellington, New Zealand. 2013. Available at: https://psychology.victoria.ac.nz/modgraph/. Accessed February 13, 2020.

35. Kraemer KL, Mayo-Smith MF, Calkins DR. Independent clinical correlates of severe alcohol withdrawal. Subst Abus 2003;24:197-209.

36. Webb GR, Redman S, Hennrikus D, Rostas JA, Sanson-Fisher RW. The prevalence and sociodemographic correlates of high-risk and problem drinking at an industrial worksite. Br J Addict 1990;85:495-507.

37. Richter K, Peter L, Rodenbeck A, Weess HG, Riedel-Heller SG, Hillemacher T. Shiftwork and alcohol consumption: a systematic review of the literature. Eur Addict Res 2021;27:9-15.

38. Noël X, Van der Linden M, d'Acremont M, Bechara A, Dan B, Hanak $\mathrm{C}$, et al. Alcohol cues increase cognitive impulsivity in individuals with alcoholism. Psychopharmacology 2007;192:291-298.

39. Sharma D, Albery IP, Cook C. Selective attentional bias to alcohol related stimuli in problem drinkers and non-problem drinkers. Addiction 2001;96:285-295.

40. Lusher J, Chandler C, Ball D. Alcohol dependence and the alcohol Stroop paradigm: evidence and issues. Drug Alcohol Depend 2004; 75:225-231.

41. Baune BT, Ponath G, Rothermundt M, Riess O, Funke H, Berger K. Association between genetic variants of IL-1beta, IL-6 and TNF-alpha cytokines and cognitive performance in the elderly general population of the MEMO-study. Psychoneuroendocrinology 2008;33:68-76.

42. Lloyd-Jones DM, Liu K, Tian L, Greenland P. Narrative review: assessment of C-reactive protein in risk prediction for cardiovascular disease. Ann Int Med 2006;145:35-42.

43. Bower JK, Lazo M, Juraschek SP, Selvin E. Within-person variability in high-sensitivity C-reactive protein. Arch Int Med 2012;172:1519-1521.

44. Roberts WL. CDC/AHA Workshop on Markers of Inflammation and Cardiovascular Disease: Application to Clinical and Public Health Practice: laboratory tests available to assess inflammation--performance and standardization: a background paper. Circulation 2004;110:e572e576.

45. Riccio CA, Reynolds CR, Lowe P, Moore JJ. The continuous performance test: a window on the neural substrates for attention? Arch Clin Neuropsychol 2002;17:235-272.

46. Pedraz-Petrozzi B, Neumann E, Sammer G. Pro-inflammatory markers and fatigue in patients with depression: a case-control study. Sci Rep 2020;10:9494.

47. Toker S, Shirom A, Shapira I, Berliner S, Melamed S. The association between burnout, depression, anxiety, and inflammation biomarkers: C-reactive protein and fibrinogen in men and women. J Occup Health Psychol 2005;10:344-362.

48. Shirom A, Melamed S. A comparison of the construct validity of two burnout measures in two groups of professionals. Int J Stress Manag 2006;13:176-200.

49. von Känel R, Bellingrath S, Kudielka B. Association between burnout and circulating levels of pro- and anti-inflammatory cytokines in schoolteachers. J Psychosom Res 2008;65:51-59.

50. Passos IC, Vasconcelos-Moreno MP, Costa LG, Kunz M, Brietzke E, Quevedo J, et al. Inflammatory markers in post-traumatic stress disorder: a systematic review, meta-analysis, and meta-regression. Lancet Psychiatry 2015;2:1002-1012. 